Le Lait (1985), 65 (1), 65-84

\title{
Mise au point d'un fermenteur de laboratoire grâce à un micro-ordinateur de faible coût
}

\author{
par \\ J. TAYEB, J. E. LEFEVRE, C. BOUILLANNE \\ et M. J. DESMAZEAUD* \\ Avec la collaboration technique de Michèle LANDON
}

\section{R és u m é}

Cette note montre qu'il est possible de compléter un fermenteur de laboratoire par un micro-ordinateur de faible coût du type " ordinateur individuel » complété par des interfaces du commerce. Le calculateur contrôle la température, le pH, l'oxygène dissous, la vitesse d'agitation, un apport de nutriments et l'échantillonnage. Il remplit les fonctions de superviseur et de stockage, de tracé de courbes et de traitement mathématique, et gère le fichier d'expérience.

Deux applications sont présentées : étalonnage universel d'une sonde à oxygène dissous et mesure dynamique du kı d'un fermenteur.

Mots clés

Fermentation - Microinformatique - Automatisation - Oxygène dissous.

\section{S u m m a r y}

SETTING UP OF A LABORATORY COMPUTERIZED BIOREACTOR

This publication describes a computerized bioreactor combining conventional regulation systems and the possibilities of a low cost personal computer with additional commercial interfaces. The

* I.N.R.A./C.N.R.Z., Laboratoire de Microbiologie Laitière - 78350 Jouy-en-Josas (France). 
computer controls temperature, $\mathrm{pH}$, dissolved oxygen, agitation speed, nutrient supplies and sampling. It fulfills the functions of set-point controlling, data storage, curve plotting, mathematical processing and manages the experimental file.

The connection of a bioreactor to a computer not only allows a precise knowledge of the environmental conditions but also opens a large field of possibilities, including the constitution of a data bank, on-line and off-line calculations and feedback control. We show here that the evolution of instrumentation, electronic and computer techniques, combined with the special characteristics of fermentation, enable a laboratory bioreactor to be upgraded by a personal computer.

The choice of the computer should be based primarily on the criteria of rapidity, memory capacity, reliability and possibilities of interfacing. Configuration as a set-point controller for monitoring fermentation experiments imposes few restrictions on the first three points. Computer connection is more and more designed into fermentation material, and some microcomputers possess multiple plug-in boards.

The software is written in BASIC with a few subroutines in machine langage. The originality of the on-line program resides in a mixed structure between a synchronous interruption program and the asynchronous main program. This simple structure enables the 16 channels to be read, calculations to be performed, measurements to be displayed and curves to be updated every two seconds, which is better than needed for fermentation experiment.

The main purpose of the bioreactor is the control of the growth of mixed culture of lactic acid bacteria. But we present two applications dealing with oxygen wich are related to controlling the superficial flora of cheese:

- for calibration of dissolved oxygen probe, the classical way (in \% of saturation) is far from being perfect since the quantity of dissolved oxygen at saturation depends on temperature, pressure and composition of the medium. An absolute and reproductible calibration (in $m g \mathrm{O}_{2} / l$ of solution) can be obtained by the use of a very simple program. All that is required is to enter the temperature and the composition of the medium;

- the gas-liquid transfer coefficient $k_{l} a$ is part of the basic characteristics of an installation. It may be measured with chemical or enzymatic methods, or by a static or a dynamic method with a dissolved oxygen probe. The program used allows dynamic calculation of $k_{l} a$ during fermentation.

Key words

Fermentation - Microcomputer - Automatisation-dissolved oxygen. 


\section{INTRODUCTION}

La recherche de haut niveau en fermentation s'intéresse actuellement à la description mathématique des processus et au développement des techniques d'optimisation (Humphrey, 1977; Armiger, 1978 ; Hampel, 1979; Unden et coll., 1979 ; Blachère, 1980 ; Sevely et Pourciel, 1980; Verès et coll., 1981; Nyeste et coll., 1981; Nanba et coll., 1981 ; Rolf, 1982 ; Rolf et Henry, 1982 ; Bernard et coll., 1983). Même si l'on ne désire pas appliquer de techniques mathématiques complexes, le suivi des expériences de fermentation pose un certain nombre de problèmes :

- la sensibilité des réactions biologiques aux conditions de milieu (température, $\mathrm{pH}$, composition, etc.) suppose une connaissance exacte de l'historique d'une fermentation;

- les capteurs actuellement disponibles ne permettent l'accès à des paramètres représentatifs des processus biochimiques qu'en combinant par le calcul leurs mesures (Humphrey, 1977; Wang et coll., 1977, 1979; Armiger, 1978; Swartz, 1978; Hampel, 1979; Unden et coll., 1979). De plus, ces capteurs sont souvent eux-mêmes sensibles aux conditions de milieu (Corrieu, 1984);

- l'application des théories existantes est freinée par la mauvaise qualité des mesures et par les calculs complexes souvent nécessaires ;

- enfin, la durée des expériences de cultures de micro-organismes implique une surveillance $24 \mathrm{~h}$ sur 24 pour échantillonner, faire un apport de nutriment, ou modifier les conditions de culture.

La connexion d'un fermenteur à un ordinateur permet non seulement de résoudre ces problèmes (Rolf et Henry, 1982), mais ouvre de plus un très grand nombre de perspectives : constitution d'une banque de données mises en forme, calculs en temps réel [quotient respiratoire, taux de croissance, rendements (Waithe et coll., 1978; Yano et coll., 1981)] et en différé, contrôle en retour (échantillonnage, apport exponentiel de nutriments, étalonnage des sondes, test de perturbation, suivi des courbes d'optimisation). Cette possibilité de contrôle en retour, rarement signalée, est une des caractéristiques originales de l'installation que nous décrivons.

La seconde originalité est l'utilisation d'un micro-ordinateur de type ordinateur individuel qui a été rendue possible grâce à l'évolution des techniques en instrumentation, électronique et informatique, associée aux caractéristiques particulières de la fermentation. Cette solution présente l'avantage d'être peu coûteuse; elle peut s'appliquer dans les nombreux domaines de recherche où se posent des problèmes de suivi et de contrôle automatique en laboratoire.

Nous développerons deux exemples caractéristiques : étalonnage d'une sonde à oxygène dissous et mesure dynamique du $\mathrm{k}_{1} \mathrm{a}$. Un 
troisième exemple pourra être trouvé dans une étude consacrée à l'optimisation d'une culture mixte de bactéries lactiques (Tayeb et coll., 1984).

\section{MATERIEL ET PROGRAMMES}

\section{Choix du matériel informatique}

L'informatisation d'une chaîne de mesure peut se faire de deux façons. Le calculateur peut se contenter de fixer les points de consigne : on parle de calculateur superviseur (Set Point Control) (fig. 1). Il peut aussi remplacer le régulateur : on parle de commande directe (Digital Direct Control) (fig. 2).

Les avantages principaux de montage DDC sont une interactivité entre boucles plus facile, une régulation pouvant être très fine par l'emploi d'algorithmes évolués, la possibilité de connaître les corrections (ce qui renseigne sur l'évolution du processus) et une plus grande souplesse d'adaptation. Les avantages principaux du montage en superviseur sont une réalisation pratique plus aisée, une sécurité plus facile à réaliser, une possibilité de fonctionner de façon classique, des programmes beaucoup plus simples et enfin des exigences restreintes au niveau performances du matériel informatique. C'est cette solution que nous avons adoptée et qui sera décrite dans ce qui suit.

Le choix du matériel informatique doit se faire principalement sur les critères rapidité, capacité mémoire, fiabilité, possibilité d'interfaçages. Le montage en calculateur superviseur pour suivre des expériences de fermentation impose des contraintes faibles sur les trois premiers points. Les phénomènes sont lents et continus : les temps caractéristiques sont de l'ordre de $5 \mathrm{~min}$ en période d'acquisition, et de quelques dizaines d'heures en durée; si on désire étudier des phénomènes transitoires, en modélisation par exemple, les durées seront courtes, et on pourra se consacrer totalement à l'acquisition. La capacité mémoire doit être suffisante pour programmer, mais n'est pas critique en stockage car on peut accéder aux mémoires de masse entre les mesures. Pour la fiabilité, le montage en superviseur et le fait de travailler à l'échelle laboratoire impliquent qu'une panne n'a pas de conséquences catastrophiques, au pire un essai à refaire. L'ensemble de ces considérations permet de penser qu'un ordinateur individuel de grande diffusion est tout à fait suffisant, sous réserve de posséder de bonnes possibilités d'interfaçage.

\section{Interfaçage et connexions}

En acquisition, on dispose généralement au niveau du matériel de mesure de sorties analogiques qui peuvent être branchées direc- 


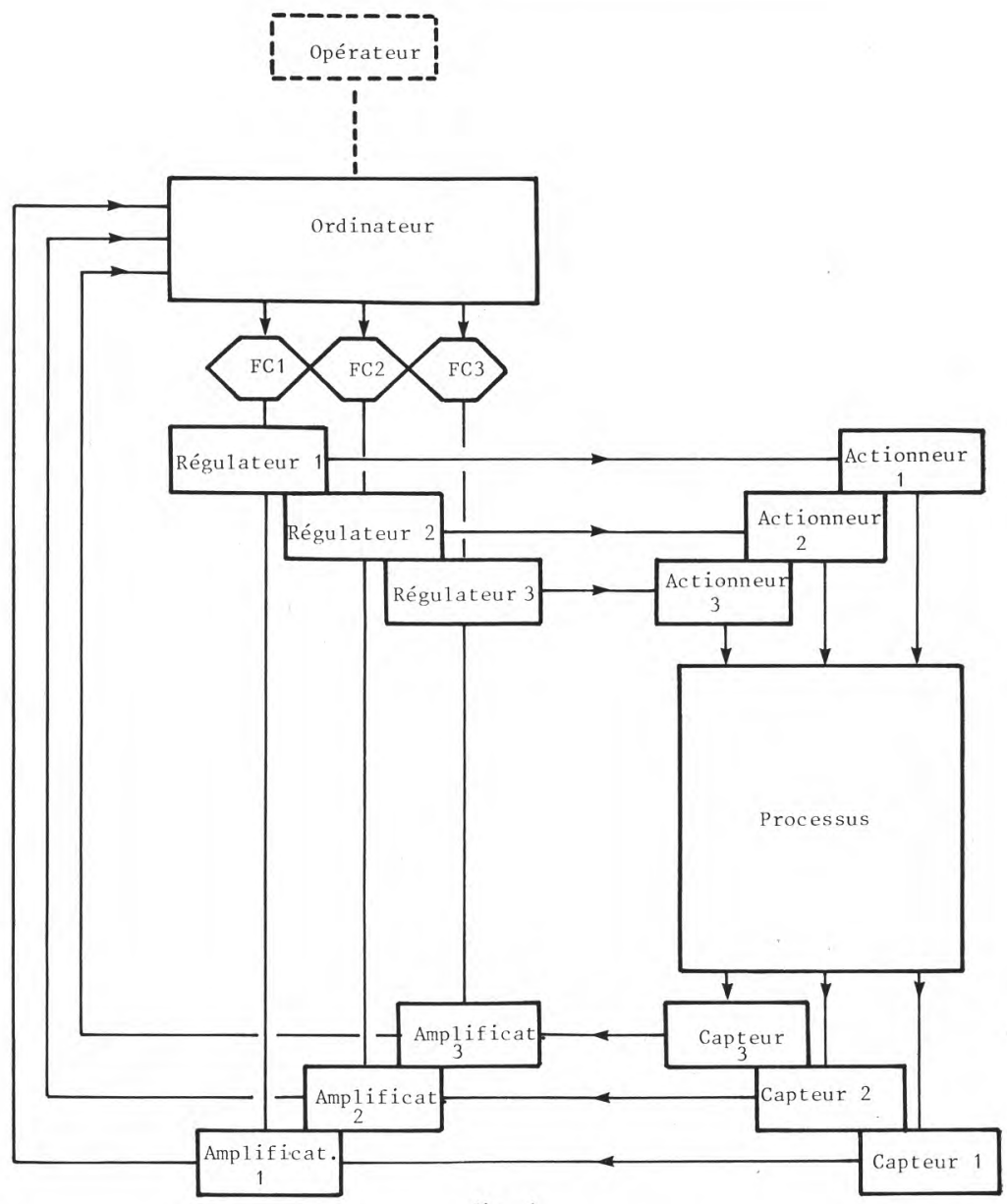

fig. 1

Schéma d'un système en calculateur superviseur. FC : Dispositif de fixation du point de consigne.

Diagram of Multiparameters Direct Set Point Control. FC : Set Point device.

tement sur une carte glissée dans l'ordinateur et comportant un multiplexeur et un convertisseur analogique/digital. S'il n'y a pas de sortie ou s'il se pose des problèmes d'isolation, un circuit intégré du type «amplificateur d'instrumentation » permet de résoudre le problème. L'adoption d'un standard unique en courant pour tous les capteurs (tel le 4-20 mA) facilite grandement la conception du système. 


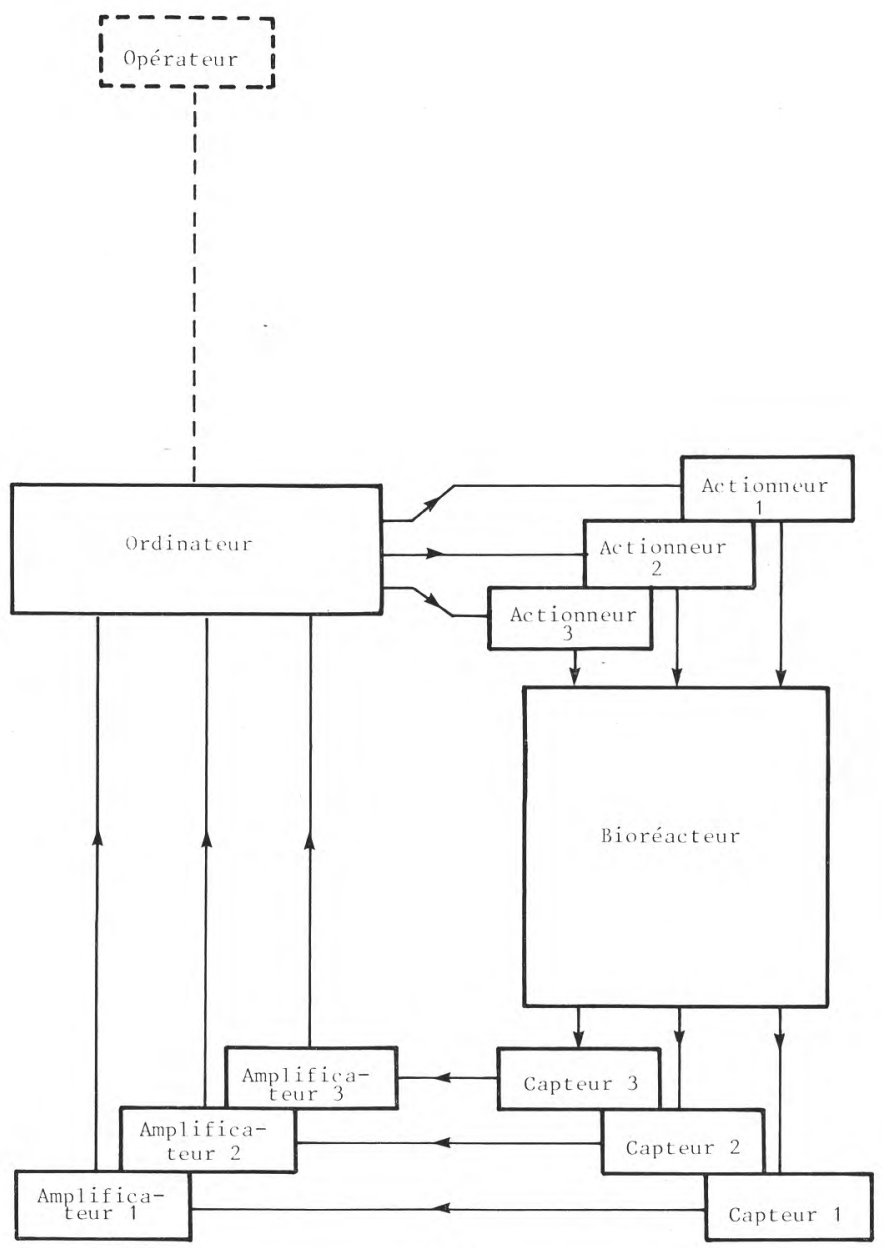

fig. 2

Schéma d'un système en contrôle digital direct.

Diagram of Multiparameters Digital Direct Control.

Pour la commande du point de consigne les régulateurs modernes disposent d'une entrée de commande à brancher sur une carte de conversion digital/analogique, éventuellement isolée par un amplificateur d'instrumentation. S'il n'y a pas d'entrée, on peut la créer à la place du potentiomètre de réglage manuel ou, plus simplement, motoriser ce potentiomètre à l'aide d'un moteur pas à pas commandé par la carte correspondante. 
Pour le contrôle des divers accessoires en tout ou rien (échantillonneur, vannes, pompes) il suffit de brancher des relais statiques à une carte d'entrées/sorties digitales à usage général. On peut ainsi commander jusqu'à 25 A sans risque de parasites.

Il faut signaler qu'il est indispensable de vérifier la comptabilité matérielle et logicielle des différentes cartes d'interfaces.

\section{Réalisation pratique}

Le fermenteur (Biolafitte) de capacité 151 est équipé des régulations de vitesse moteur, température, $\mathrm{pH}$ et $\mathrm{O}_{2}$ dissous (Biolafitte). Il est complété par un dispositif pour addition quantitative

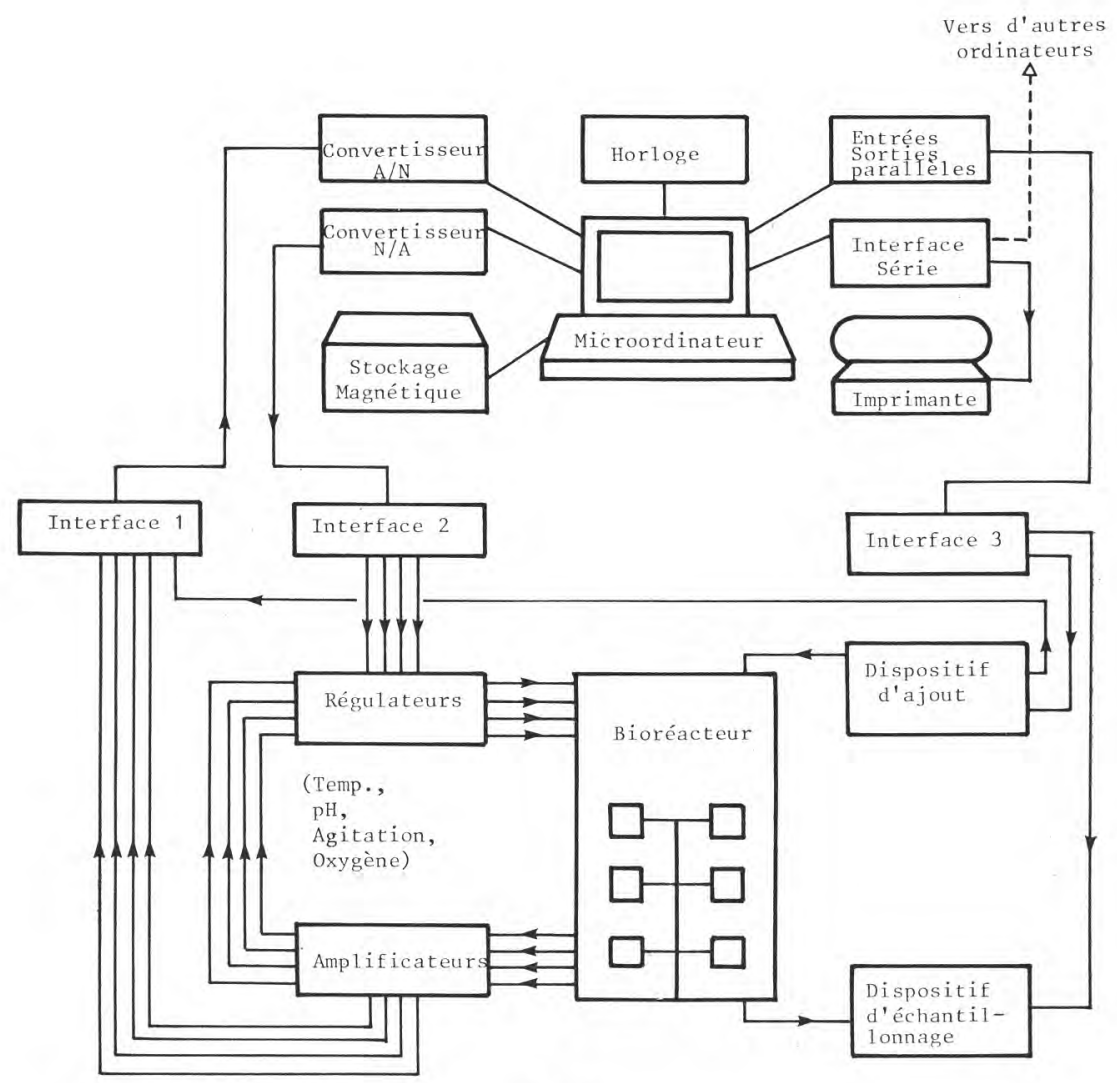

fig. 3

Schéma du bioréacteur de laboratoire informatisé. Diagram of th? Computerized Laboratory Fermentor. 
de substrat (Biolafitte) et par un échantillonneur automatique stérile réfrigéré (Braun).

La gestion de l'ensemble est confiée à un micro-ordinateur Apple II $48 \mathrm{~K}$ octets (Apple Computer Inc.) muni d'une imprimante et de deux mini-disques de mémoires de masse. Le micro-ordinateur est complété par les cartes suivantes : carte de liaison série RS 232 (California Computer System) pour connexion de l'imprimante et

Interface 1: transformation de courant en tension pour 1'acquisition des données.

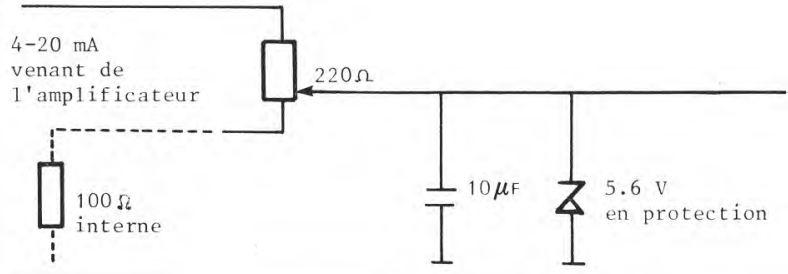

Interface 2 : Contrôle du point de consigne par l'ordinateur.

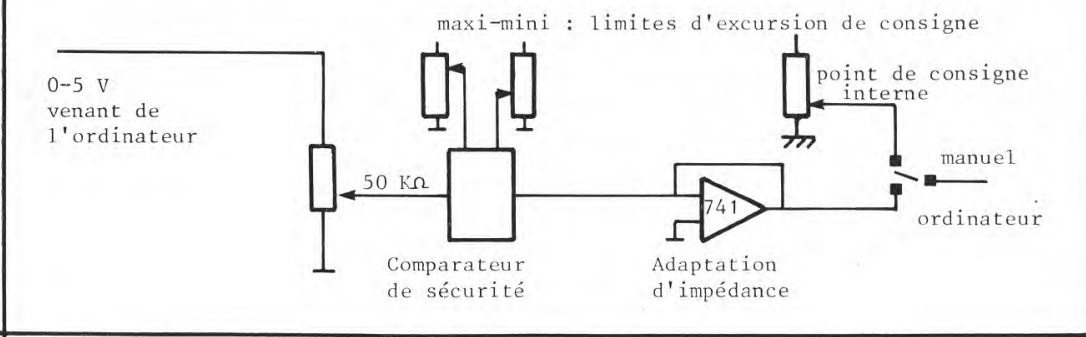

Interface 3 : Sorties tout ou rien

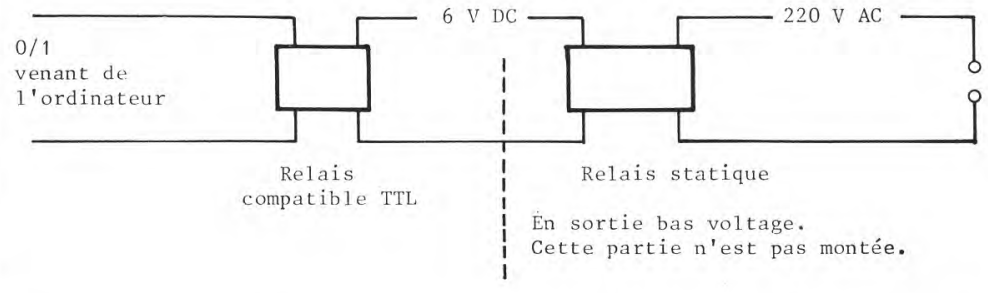

fig. 4

Interfaces entre le matériel de fermentation et l'ordinateur.

Interfaces between Computer Cards and Fermentation Equipment. 
vers le centre de calcul, carte horloge (California Computer System), carte d'acquisition analogique/digital 16 voies, 12 bits, 3 gammes (MID Paris), carte de conversion digital/analogique 4 voies, 12 bits, 3 gammes (MID Paris), carte 8 entrées/8 sorties digitales (MID Paris). L'ensemble informatique est protégé par un onduleur commuté (IEF Paris). L'ensemble est présenté en figure 3.

L'électronique de base Biolafitte permet l'acquisition de données mais pas la commande de points de consigne. Nous avons réalisé les interfaces schématisées en figure 4 pour le branchement en acquisition, la commande des points de consigne et la commande en tout ou rien. Le travail sur 12 bits permet une résolution théorique de $0,04^{\circ} \mathrm{C}$ ou 0,003 unité $\mathrm{pH}$, bien meilleure que celle de la sonde elle-même.

Les prix de revient du matériel informatique, d'acquisition et de commande est d'environ $40000 \mathrm{FF}(5000$ \$). Cela ne représente que 15 à $20 \%$ du prix d'une installation de fermentation de laboratoire de taille moyenne mais multiplie considérablement ses possibilités et permet de l'utiliser beaucoup plus fréquemment et avec plus de profit.

\section{Programmation}

La programmation est faite en BASIC sous DOS 3.3. Ce langage est relativement lent, mais nous avons montré qu'il n'y a pas de problème critique de vitesse. Quelques sous-programmes de gestion des interfaces sont écrits en langage machine.

On peut distinguer 3 classes de fonction dans les programmes:

- la fonction "superviseur" : gestion des capteurs (acquisition de données) et gestion des régulateurs (modification des points de consigne);

- la fonction « calcul et traitement " : calcul des paramètres synthétiques, moyenne, filtrage numérique et tracé de courbes;

- la fonction " archive ": stockage des données, mise à jour de fichiers, relecture et exploitation.

Un résumé de l'organisation logique du programme temps réel apparaît figure 5 . Son originalité réside dans une structure mixte entre un programme d'interruption synchrone et un programme principal asynchrone. La partie "superviseur " est exécutée à partir d'interruptions d'horloge pour obtenir des points régulièrement espacés, et pour pouvoir modifier les paramètres du programme principal même pendant une expérimentation sans perdre d'informations. Les autres fonctions temps réel ( calcul et traitement ": moyenne, affichage, impression; " archive ": stockage sur disque) sont contrôlées à partir d'un programme principal qui les accomplit séquentiellement si les indicateurs correspondants sont positionnés. 


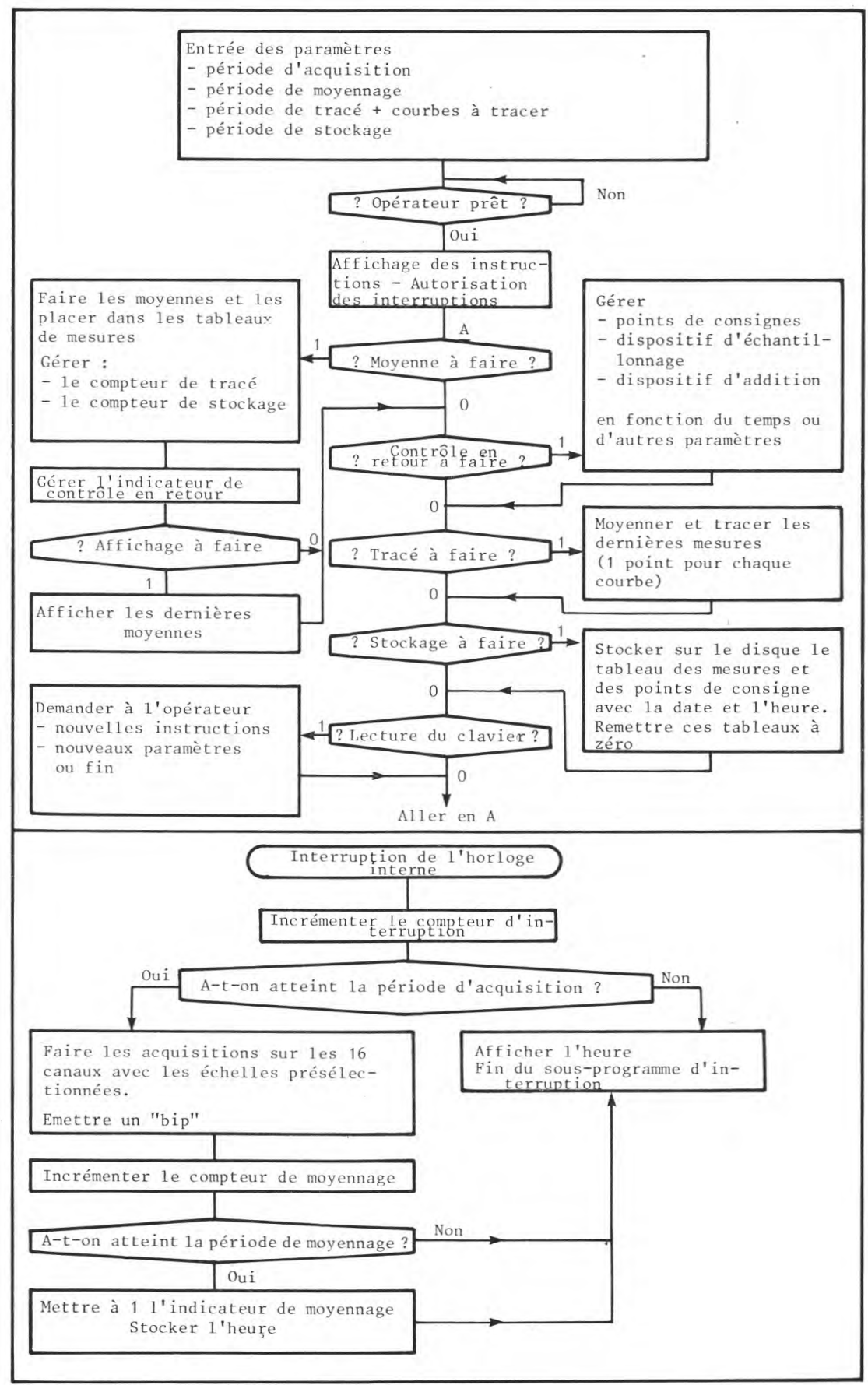

fig. 5

Organigramme du programme général en temps réel.

Flowchart of the General Purpose Real-Time Program. 


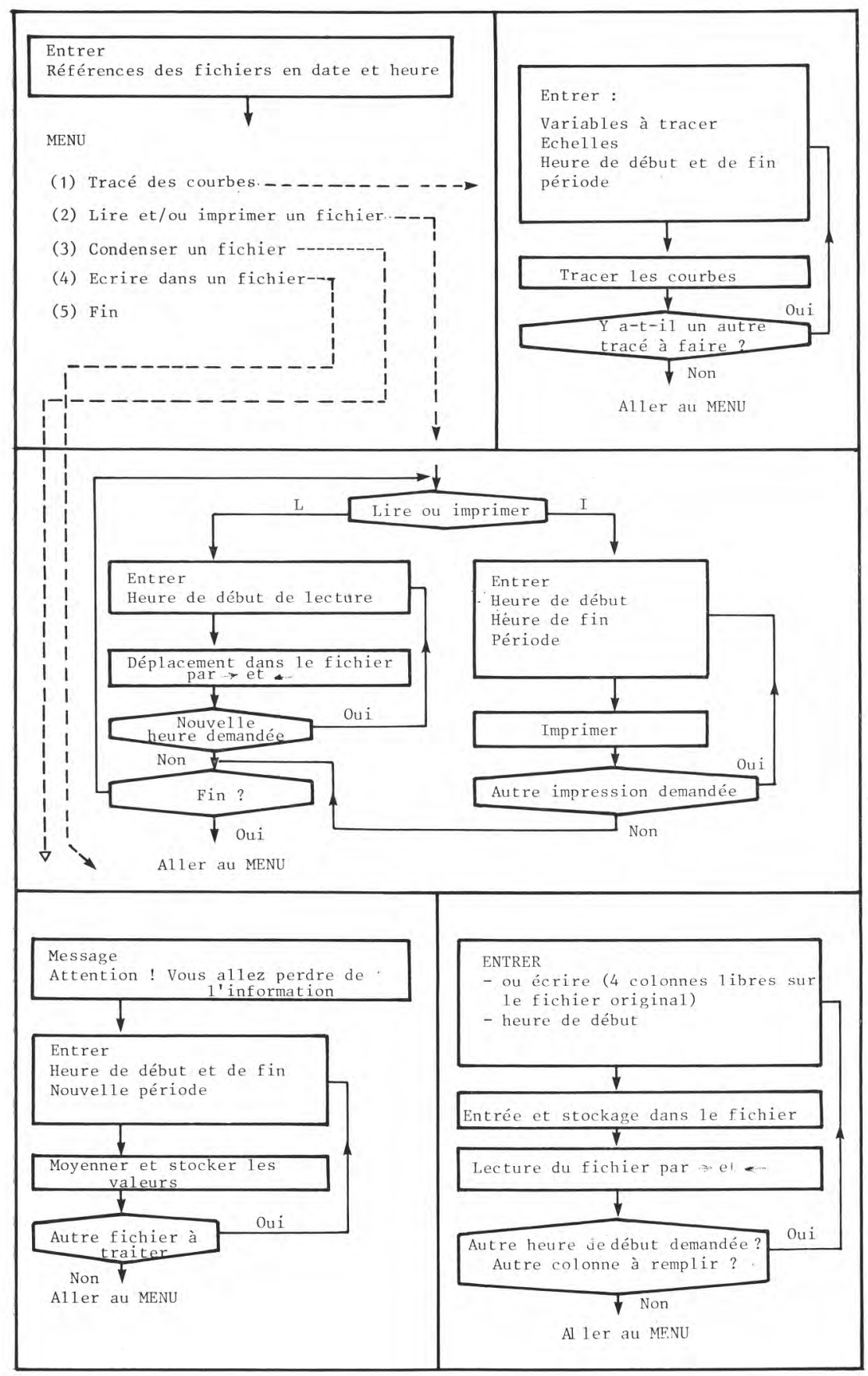

fig. 6

Organigramme du programme général d'exploitation.

Flowcharts of General Purpose Off-line Programs. 
Une structure aussi simple permet néanmoins de scruter les 16 voies, faire les calculs, afficher les mesures, imprimer les courbes toutes les 2 secondes. En pratique, pour les fermentations d'une vingtaine d'heures, on mémorise un point (moyenne de 20) toutes les minutes pour chaque voie, on complète les courbes toutes les $5 \mathrm{~min}$ et on stocke sur disque toutes les $4 \mathrm{~h}$ environ l'ensemble des informations mémorisées.

Les programmes de base exécutables en temps différé concernent la fonction " archive ". Comme le montre la figure 6 , ils permettent d'exploiter les fichiers de mesure : relecture à l'écran ou impression, tracé de courbes en modifiant les échelles, écritures de données complémentaires, compression des fichiers si l'évolution des mesures est très faible par rapport à la période choisie. Un programme annexe permet de créer, consulter et trier des fiches d'expérience comportant les caractéristiques de la souche, du milieu, du type d'expérimentation, etc.

\section{APPLICATIONS}

En complément de ses fonctions principales de stockage des mesures et de prise de décision durant la fermentation, trop longues à illustrer ici, un micro-ordinateur permet de simplifier un grand nombre des opérations annexes de la fermentation : stérilisation, étalonnage des capteurs, mesures diverses. Nous allons prendre deux exemples qui montrent que l'informatique permet l'emploi de théories complexes, grâce à la simplification qu'elle apporte.

\section{Etalonnage de la sonde $\mathrm{O}_{2}$ dissous}

La définition d'un étalonnage reproductible et universel d'une sonde à oxygène dissous est rarement abordée. L'approche classique en $\%$ de la saturation est loin d'être parfaite puisque la quantité d'oxygène dissous à saturation dépend de la température, de la pression et de la composition du milieu (Popovic et coll., 1979; Quickert et coll., 1981). Un essai à $80 \%$ à $37^{\circ} \mathrm{C}$ et à $80 \%$ à $25^{\circ} \mathrm{C}$ ne correspond donc pas à la même quantité d'oxygène dissous, exprimée en $\mathrm{mg} \mathrm{O}_{2} / 1$ de solution. Pourtant c'est cette quantité qui influence les micro-organismes.

* L'influence de la température, de la pression et de la composition du milieu dans le domaine abordé en fermentation s'écrit :

(a) $\mathrm{S}_{0}=4,90 \cdot 10^{-2}-1,335 \cdot 10^{-3} \cdot t+2,759 \cdot 10^{-5} \cdot t^{2}-3,235 \cdot 10^{-7} \cdot t^{3}$

$+1,614 \cdot 10^{-9} \cdot t^{4}$

$\mathrm{S}_{\circ}$ : solubilité de l'oxygène en $\mathrm{O}^{2} / 1$ d'eau

$t$ : température en ${ }^{\circ}$ celsius 


\section{TABLEAU 1 - TABLE 1}

Coefficients de Sechenov $\mathrm{Ki}$ et Van Krevelen Hoftizer $\mathrm{Hj}$ pour l'oxygène à $25^{\circ} \mathrm{C}$

Sechenov (Ki) and Van Krevelen Hoftizer $(\mathrm{H} j)$ coefficients for oxygen at $25^{\circ} \mathrm{C}$

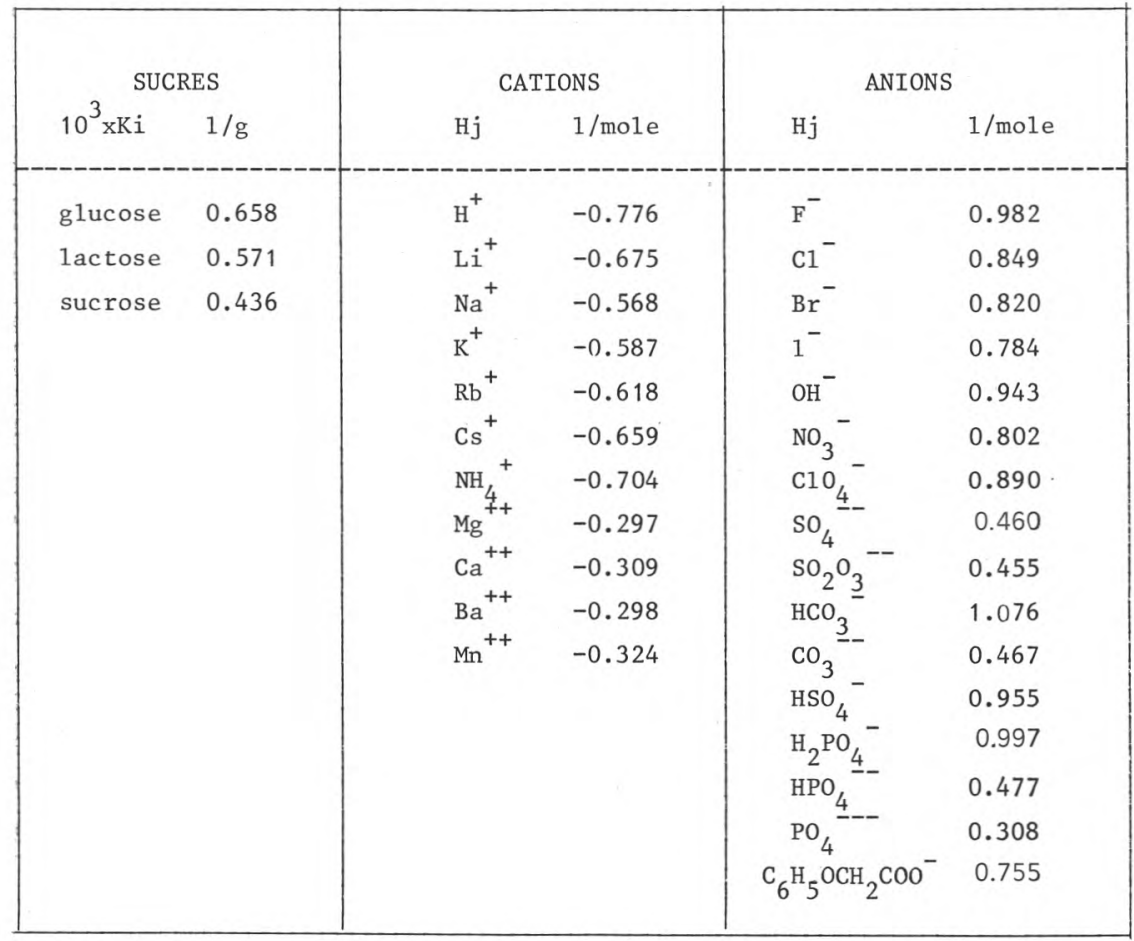

(b) $\mathrm{S}=\mathrm{S}_{o} \frac{\mathrm{P}}{\mathrm{P}_{\mathrm{o}}}$ (Loi de Henry)

(c) $\log \frac{\mathrm{S}_{o}}{\mathrm{~S}}=\underset{\mathrm{i}=1}{\mathrm{P}} \mathrm{K}_{\mathrm{i}} \mathrm{C}_{\mathrm{i}}$ (formule de Sechenov)

$\mathrm{K}_{1}$ : coefficient qui dépend du gaz et du sucre i (Table 1)

$\mathrm{C}_{\mathrm{i}}$ : concentration du sucre $\mathrm{i}$ dans la solution en $\mathrm{g} / \mathrm{l}$

(d) $\log \frac{\text { So }}{\mathrm{S}}=\frac{1}{2} \stackrel{\mathrm{P}}{\mathrm{j}}=1 \quad \mathrm{H}_{\mathrm{j}} \mathrm{C}_{\mathrm{j}} \mathrm{Z}_{\mathrm{j}}^{2}$ (Formule de Schumpe, 


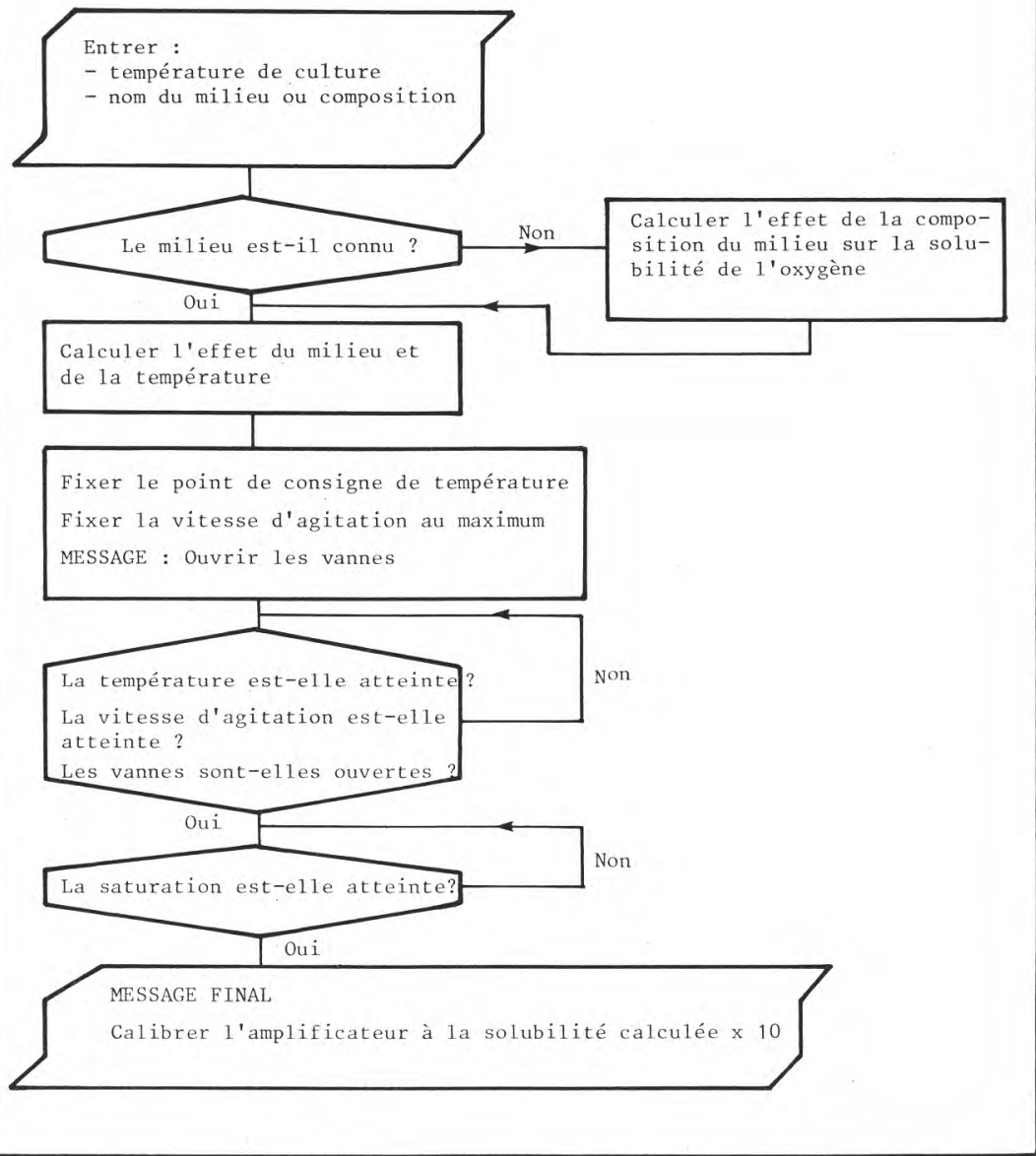

fig. 7

Organigramme du programme d'étalonnage de la sonde à oxygène dissous.

Flowchart of the Dissolved Oxygen Probe Calibration Program.

\section{Adler et Deckwer)}

$\mathrm{H}_{\mathrm{j}}$ : coefficient qui dépend du gaz et de l'ion $\mathrm{j}$ (table 1)

$\mathrm{C}_{\mathrm{j}}$ : concentration de l'ion $\mathrm{j}$ dans la solution en mole/1

$\mathrm{Z}_{\mathrm{j}}$ : valence de l'ion $\mathrm{j}$

A partir de ces formules, on peut calculer la solubilité de l'oxygène à saturation pour une température, une pression et une compo- 
sition de milieu données. Dans ces conditions, après avoir atteint la saturation, on peut calibrer la sonde par rapport à la valeur calculée et obtenir une mesure absolue et universelle de la concentration en oxygène dissous.

L'informatisation du fermenteur apporte une solution élégante à l'étalonnage en $\mathrm{mg} \mathrm{\textrm {O } _ { 2 }} / \mathrm{l}$ grâce à un programme très simple comme celui de la figure 7 . Il suffit d'entrer la température de la culture et le nom du milieu, ou la composition si celui-ci n'est pas référencé et d'ouvrir les vannes lorsque le message apparaît. On obtient ainsi un étalonnage universel et reproductible de la sonde en $\mathrm{mg} \mathrm{\textrm {O } _ { 2 } / 1}$ de solution.

\section{Mesure du $K_{1}$ a par méthode dynamique}

Le $K_{1}$ a est le coefficient de transfert gaz-liquide pour l'oxygène. Il dépend des variables technologiques : géométrie du fermenteur, pression et vitesse des gaz injectés, température, vitesse d'agitation, viscosité du milieu. Il fait partie des caractéristiques de base d'une installation et influence indirectement les processus microbiologiques par le biais des gaz dissous (oxygène ou sous-produits du métabolisme).

On peut le mesurer (Kappeli et Fiechter, 1980; Spriet et coll., 1982) par des méthodes chimiques ou enzymatiques : méthode au sulfite, à l'hydrazine, ou à la glucose oxydase. L'inconvénient de ces méthodes est que l'on ne travaille pas dans les conditions de culture. On peut aussi le mesurer avec une sonde à oxygène dissous, par deux méthodes. La méthode statique se base sur la courbe obtenue lors du passage d'une concentration en oxygène nulle (saturation en azote) à la saturation dans les conditions expérimentales étudiées mais sans micro-organismes. La méthode dynamique s'emploie durant la fermentation ; on est certain d'être alors dans les conditions de culture.

Durant la fermentation, on a : $\frac{\mathrm{dC}_{1}}{\mathrm{dt}}=\mathrm{K}_{1 \mathrm{a}}\left(\mathrm{CX}-\mathrm{C}_{\mathrm{I}}\right)-\mathrm{V}_{\mathrm{r}} \mathrm{X}$

$C^{*}$ : concentration à saturation

$\mathrm{C}_{1}$ : concentration au temps $\mathrm{t}$ mesurée par la sonde

$\mathrm{V}_{\mathrm{r}}$ : vitesse de respiration des micro-organismes

$\mathrm{X}$ : concentration des micro-organismes

Si on coupe l'agitation et l'aération, il n'y a plus de transfert, d'où : $\frac{\mathrm{dC}_{1}}{\mathrm{dt}}=-\mathrm{V}_{\mathrm{r}} \mathrm{X}$

donc l'enregistrement du signal fournit une droite de pente $-\mathrm{V}_{\mathrm{r}} \mathrm{X}$. 


\section{TABLEAU 2 - TABLE 2}

Détermination dynamique du $\mathrm{K}_{1} \mathrm{a}$

DO : Valeur de l'oxygène dissous ( $\%$ de la saturation).

Agitation : 200 tpm. Aération : débit d'air $15 \mathrm{l} / \mathrm{min}$.

Débit d'azote 15 1/min. Brevibacterium linens CNRZ 906.

Dynamic Determination of $K_{1} a$

DO : Dissolved Oxygen value ( $\%$ of saturation).

Agitation : $200 \mathrm{rpm}$. Aeration : Air Flow Rate $=15 \mathrm{l} / \mathrm{min}$, Nitrogen.

Flow rate $=15 \mathrm{l} / \mathrm{min}$. Brevibacterium linens CNRZ 906 .

\begin{tabular}{|c|c|c|c|c|}
\hline TEMPS & $\mathrm{D} 0_{1}$ & & & CALCULS \\
\hline $\begin{array}{r}0 \\
20 \\
40 \\
60 \\
80 \\
100 \\
120\end{array}$ & $\begin{array}{l}70.0 \\
68.4 \\
61.8 \\
54.0 \\
46.4 \\
39.5 \\
33.0\end{array}$ & & & $\begin{array}{l}\longrightarrow \mathrm{DO}_{0}=70 \% \\
\text { Régression } 1 \text { inéaire sur } \mathrm{DO}_{1} \\
\text { en fonction du temps : } \\
\mathrm{DO}_{1}=-0.329 \mathrm{~T}+73.04 \\
\text { correlation }=.993 \\
\rightarrow \operatorname{VrX}=.329 \% \mathrm{~s}^{-1}\end{array}$ \\
\hline TEMPS & DO & $\mathrm{z}$ & $\mathrm{Y}$ & \\
\hline 140 & 36.9 & 33.1 & - & \\
\hline 160 & 40.0 & 30.0 & 0.492 & \\
\hline 180 & 43.4 & 26.6 & 0.489 & Régression linéaire \\
\hline 200 & 46.4 & 23.6 & 0.484 & $\mathrm{DO}_{2}(\mathrm{~T}+1)-\mathrm{DO}_{2}(\mathrm{~T}-1)$ \\
\hline $\begin{array}{l}220 \\
240\end{array}$ & $\begin{array}{l}49.6 \\
52.4\end{array}$ & $\begin{array}{l}20.4 \\
17.6\end{array}$ & $\begin{array}{l}0.479 \\
0.454\end{array}$ & $\operatorname{sur} Y=-\underbrace{T} T^{+}$ \\
\hline 260 & 54.6 & 15.4 & 0.439 & \\
\hline 280 & 56.8 & 13.2 & 0.424 & en fonction de $\mathrm{Z}=\mathrm{DO}_{0}-\mathrm{DO}_{2}(\mathrm{~T})$ \\
\hline 300 & 58.4 & 11.6 & 0.409 & 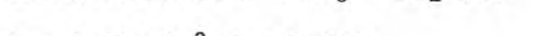 \\
\hline 320 & 60.0 & 10.6 & 0.399 & $Y=5.3910^{-3} \mathrm{Z}+0.350$ \\
\hline 340 & 61.2 & 8.8 & 0.394 & \\
\hline 360 & 62.6 & 7.4 & 0.394 & correlation $=.985$ \\
\hline 380 & 63.8 & 6.2 & 0.379 & \\
\hline 400 & 64.6 & 5.4 & 0.369 & \\
\hline 420 & 65.4 & 4.6 & 0.369 & $\longrightarrow \mathrm{K}_{1} \mathrm{a}=5.3910^{-3} \mathrm{~s}^{-1}$ \\
\hline 440 & 66.2 & 3.8 & 0.369 & 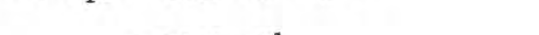 \\
\hline 460 & 67.0 & 3.0 & 0.369 & $=19.4 \mathrm{~h}^{-1}$ \\
\hline 480 & 67.8 & 2.2 & 0.369 & \\
\hline 500 & 68.6 & 1.4 & 0.359 & \\
\hline 520 & 60.0 & 1.0 & 0.349 & \\
\hline
\end{tabular}

On rétablit ensuite l'aération puis on trace $\frac{\mathrm{dC}_{1}}{\mathrm{dt}}+\mathrm{V}_{\mathrm{r}} \mathrm{X}$ en fonction de $\mathrm{C}^{*}-\mathrm{C}_{1}$ et on obtient une droite de pente $\mathrm{K}_{1} \mathrm{a}$. (Le tableau 2 donne un exemple de traitement mathématique manuel des mesures). 


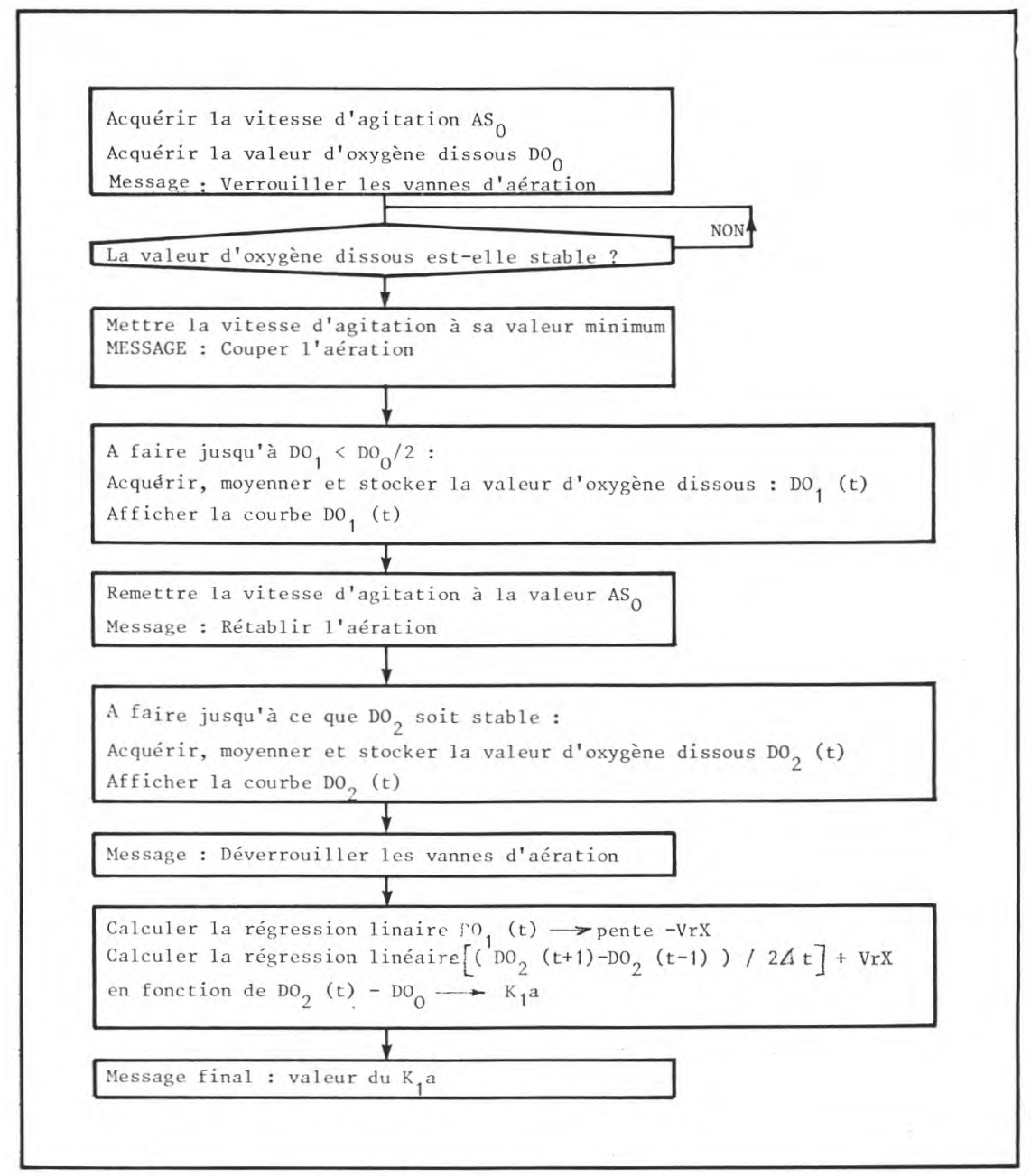

fig. 8

Organigramme du programme de calcul dynamique du $\mathrm{K}_{1} \mathrm{a}$.

Flowchart of Dynamic $K_{1}$ a Calculation Program.

La micro-informatique permet de multiplier les mesures dynamiques de $\mathrm{K}_{1}$ a grâce à la simplicité de la manipulation. Le programme représenté figure 8 prend en charge l'ensemble des opérations et des calculs. Les seules interventions de l'opérateur sont l'ouverture et la fermeture des vannes lorsque le calculateur le demande. 


\section{CONCLUSION}

Dans l'avenir, l'informatique permettra de prendre en compte l'information des nombreux capteurs (telles les électrodes enzymatiques) nécessitant des calculs en ligne (Kernevez et coll., 1983). De plus, la connexion (prévue sur notre système) du micro-ordinateur d'expérimentation sur un gros ordinateur commun à tout un centre de recherches permettra de mettre en œuvre les techniques de modélisation et d'optimisation. (Youssef-Pour et Williams, 1981 ; Dairaku et coll., 1982 ; Staniskis et Levisauskas, 1983).

\section{Remerciements}

Les auteurs remercient Mlle Marie-Hélène Famelart et M. Jean Hermier pour les conseils et les discussions qui ont permis la réalisation de ce travail.

Une partie de ce travail a bénéficié d'une aide du Ministère de la Recherche et de l'Industrie : Contrat $\mathrm{n}^{\circ}$ T.A.A. 81/C. 1298/046.

\section{Remarque}

Les détails strictement techniques de la réalisation sont disponibles auprès des auteurs.

\section{Bibliographie}

ARMiger (W.B.) (1978). - Computer applications in fermentation technology. Biotechnol. Bioeng. Symp. $\mathrm{n}^{\circ}$ 9, $398 \mathrm{p}$.

Bernard (A.), Cordonnier (M.), Lebeault (J.M.) (1983). - A DDC pilot plant system for fermentation control. Process Biochem., 18, 3, 2-5.

BLACHÈRE (H.) (1980). - Perspectives nouvelles pour le contrôle et la conduite optimale dans les procédés biotechnologiques. In : Fermentation : réacteurs, capteurs et automatisation, 253-262. Blachère et Durand (éd.), Société Française de Microbiologie, Paris.

CoRrIEU (G.) (1984). - Les capteurs dans les industries agro-alimentaires. Ind. Alim. Agr., 1143-1150.

Dairaku (K.), Yamasaki (Y.), Mortkawa (H.), Shioya (S.), Takamatsu (T.) (1982). Experimental study of time-optimal control in fed-batch culture of bakers-yeast. J. Ferment. Technol., 60, 67-75.

HAMPEL (W.A.) (1979). - Application of microcomputers in the study of microbial processes. Adv. Biochem. Eng., 13, 1-33.

HUMPHREY (A.E.) (1977). - The use of computers in fermentation systems. Process Biochem., 12, 19-22, 24-25.

KAPPELI (O.), FIECHTER (A.) (1980). - Biological method for the measurement of the maximum oxygen transfer rate of a bioreactor at definite conditions. Biotechnol. Bioeng., 22, 1509-1512.

Kernevez (J.P.), Konate (L.), RometTe (J.L.) (1983). - Determination of substrate concentrations by a computerized enzyme electrode. Biotechnol. Bioeng., $25,845-855$. 
Nanba (A.), Hirota (F.), Nagai (S.) (1981). - Microputer-coupled bakers'yeast production. J. Ferment. Technol., 59, 383-389.

Nieste (L.), Szigeti (L.), Veres (A.), Pungor (E.), Kurucz (I.), Hollo (J.) (1981). Automated fermentation equipment. II. Computer-fermentor system. Biotechnol. Bioeng., 23, 405-417.

Popovic (M.), Niebelschutz (H.), Reuss (M.) (1979). - Oxygen solubilities in fermentation fluids. Eur. J. Appl. Microbiol. Biotechnol., 8, 1-15.

Quicker (G.), Schumpe (A.), Konig (B.), Deckwert (W.D.) (1981). - Comparison of measured and calculated oxygen solubilities in fermentation media. Biotechnol. Bioeng., 23, 633-650.

Rolf (M.J.), Hennigan (P.J.), Mohler (R.D.), Weigand (W.A.) (1982). - Development of a direct digital-controled fermentor using a microminicomputer hierarchical system. Biotechnol. Bioeng., 24, 1191-1210.

RolF (M.J.), HenRy (C.L.) (1982). - Computer control of fermentation processes. Enzyme Microb. Technol., 4, 370-380.

Sevely (Y.), Pourciel (J.B.) (1980). - Applications des microcalculateurs à l'étude et à la commande des procédés de fermentation. In : Fermentation : réacteurs, capteurs et automatisation, 223-252. Blachère et Durand (éd.), Société Française de Microbiologie, Paris.

Spriet (J.A.), Botterman (J.), De Buysser (D.R.), De Visscher (P.L.), Vandamme (E.J.) (1982). - A computer-aided non interfering technique for monitoring oxygen-transfer characteristics during fermentation processes. Biotechnol. Bioeng., 24, 1605-1621.

Staniskis (J.), Levisauskas (D.) (1983). - Optimization of batch fermentation processes by graphical method. Biotechnol. Bioeng., 25, 985-990.

SWARTZ (J.R.), COONEY (C.L.) (1978). - Instrumentation in computer-aided fermentation. Process Biochem., 13, 3-4, 6-7, 24.

Tayeb (J.), Boulllanne (C.), Desmazeaud (M.J.) (1984). - Computerized control of growth with temperature in a mixed culture of lactic acid bacteria. $J$. Ferment. Technol., 62, 461-470.

UNDEN (A.), Rindone (W.P.), Heden (C.G.) (1979). - The computer in fermentation process research, Process Biochem., 14, 8-10, 12.

Veres (A.), Nyestle (L.), Kurucz (J.), Kirchinopf (L.), Szigeti (L.), Hollo (J.) (1981), - Automated fermentation equipment. I. Program-controled fermentor. Biotechnol. Bioeng., 23, 391-404.

WANG (H.Y.), CoONEY (C.L.), WANG (D.I.C.) (1977). - Computer-aided bakers' yeast fermentation. Biotechnol. Bioeng., 19, 69-86.

WANG (H.Y.), COONEY (C.L.), WANG (D.I.C.) (1979). - Computer control of bakers' yeast production. Biotechnol. Bioeng., 21, 975-995.

Whaite (P.), ABorhen (S.), Hong (E.), Rogers (P.L.) (1978). - Microprocessor control of respiratory quotient. Biotechnol. Bioeng., 20, 1459-1463.

Yano (T.), Kobayashi (T.), Shimizu (S.) (1981). - Control system of dissolved oxygen concentration employing a microcomputer. J. Ferment. Technol., 59, 295-301.

Yousefpour (P.), Williams (D.) (1981), - Real time optimisation of fermentation process. Biotechnol. Lett., 3, 519. 